\title{
Estrategias para la seguridad hídrica ante los cambios de precipitación por efectos del cambio climático
}

\author{
Strategies for water security in the face of changes in precipitation due to \\ climate change effects
}

\section{Estratégias para a segurança da água em face das mudanças de precipitação devido aos efeitos das mudanças climáticas}

\author{
Sirilo Suastegui Cruz \\ Universidad Autónoma de Guerrero, México \\ Sirilo_sc@uagro.mx \\ https://orcid.org/0000-0001-6795-6312
}

\section{Resumen}

El cambio climático afecta negativamente los patrones de precipitación con breves periodos de lluvia que impactan en los ecosistemas a través de la sequía y producción de alimentos, lo que obstaculiza el suministro de agua en áreas marginadas como Las Ánimas, donde la clave de supervivencia se basa en la agricultura. Existen modelos globales de circulación como los MPI ECHAM 5 y HadGEN1 y escenarios A2 y B2 que permiten visualizar periodos de lluvias pasados y futuros para anticipar los cambios y generar estrategias desde ámbitos locales. En tal sentido, esta investigación fue de tipo cuantitativa, longitudinal-retrospectiva, no experimental y explicativa, donde se encontraron datos de la precipitación de los años 2015, 2016 y 2017 —omo los puntos más críticos 2015 y 2016 - así como las variaciones para los años 2030 y 2050, que indican que la precipitación aumentará, pero con periodos más cortos, lo cual afectará la agricultura y generara problemas de seguridad hídrica y alimentaria en zonas rurales, donde es necesario recurrir a mecanismos de resiliencia comunitaria anual con mejoras en la captación, conservación y manejo del agua.

Palabras clave: cambio climático, escenarios, estrategias, modelos, precipitación, seguridad alimentaria, seguridad hidrica, sequías. 


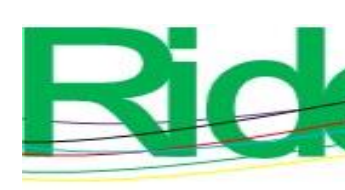

Revista Iberoamericana para la

Investigación y el Desarrollo Educativo

ISSN $2007-7467$

\section{Abstract}

Climate change negatively affects precipitation patterns, with brief periods of rainfall impacting ecosystems through drought and food production, hindering water supply in marginalized areas such as Las Animas, where the key to survival is based on the Agriculture. There are global circulation models such as the MPI ECHAM 5 and HadGEN1 and scenarios A2 and B2 that allow visualizing past and future rainy periods to anticipate changes and be able to generate strategies from local areas.

This research was quantitative, longitudinal-retrospective, non-experimental and explanatory, where precipitation data for the years 2015, 2016 and 2017 were found, as the most critical points (2015 and 2016), as well as the variations for 2030 and 2050 that indicate that precipitation will increase but with shorter periods, which will affect agriculture and generate water and food security problems in rural areas, where it is necessary to resort to annual community resilience mechanisms with improvements in catchment, conservation and water management.

Keywords: climate change, scenarios, strategies, models, precipitation, food security, water security, droughts.

\section{Resumo}

A mudança climática Afeta negativamente os padrões de precipitação, com breves períodos de chuva impactando os ecossistemas por meio da seca e da produção de alimentos, dificultando o abastecimento de água em áreas marginalizadas como Las Animas, onde a chave para a sobrevivência está baseada na agricultura. Existem modelos sistemas de circulação global como MPI ECHAM 5 e HadGEN1 e cenários A2 e B2 que permitem visualizar períodos de chuvas passados e futuros para antecipar mudanças e poder gerar estratégias a partir das áreas locais.

Esta pesquisa foi quantitativa, longitudinal-retrospectiva, não experimental e explicativa, onde foram encontrados dados de precipitação para os anos de 2015, 2016 e 2017, como os pontos mais críticos (2015 e 2016), bem como as variações para 2030 e 2050 que indicam que a precipitação aumentará mas com períodos mais curtos, o que afetará a agricultura e gerará problemas de segurança hídrica e alimentar nas áreas rurais, onde é necessário recorrer a mecanismos anuais de resiliência da comunidade com melhorias na captação, conservação e gestão da água.

Palavras chave: mudanças climáticas, cenários, estratégias, modelos, precipitação, segurança alimentar, segurança hídrica, secas. 


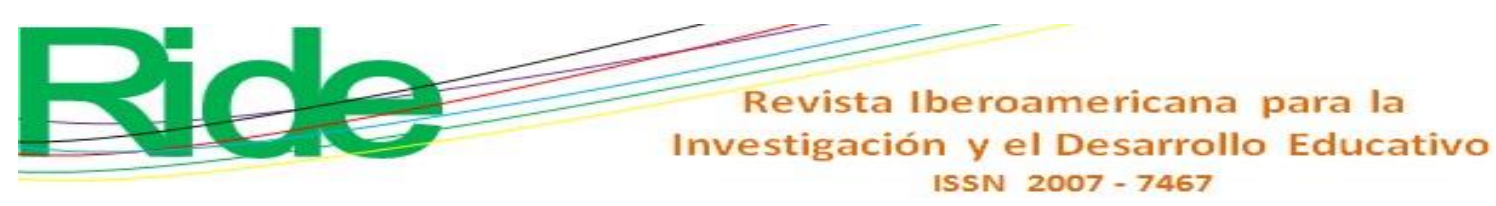

\section{Materiales y métodos}

El estudio fue de tipo cuantitativo, longitudinal-retrospectivo, no experimental y explicativo. La variable dependiente fue la cantidad de precipitación pluvial, mientras que la independiente fue los años. Como objeto de estudio se utilizaron las precipitaciones de 2015, 2016 y 2017, y se realizó el análisis de los posibles escenarios para los años 2030 y 2050 de la localidad

de Las Ánimas, municipio de Tecoanapa Guerrero (México), ubicada en las coordenadas 99 $19^{\prime}$ 07" de longitud sur y $16^{\circ} 58^{\prime} 22^{\prime \prime}$ de latitud oeste, a una altura de $660 \mathrm{~m} \mathrm{s.} \mathrm{n.} \mathrm{m.} \mathrm{El} \mathrm{clima} \mathrm{es}$ subhúmedo cálido (García, 1973) con temperatura media de $31{ }^{\circ} \mathrm{C}$, precipitación de $1200 \mathrm{~mm}$ anuales y un total de 1527 habitantes (Instituto Nacional de Estadística Geografía e Informática [Inegi], 2010).

Los datos fueron recabados del atlas climático digital de México y se obtuvo información de modelos globales de circulación (MPI ECHAM 5 y HadGEN1) y escenarios A2, B2 para los años 2030 y 2050; este tipo de modelos presentan información del territorio mexicano del cual se hicieron recortes al ráster nacional con el shapefile de Las Ánimas, con apoyo de la herramienta ArcGIS 10.3 para precipitaciones mensuales del área de estudio.

La información permitió crear bases de datos de la precipitación mensual para los años 2030 y 2050 en los escenarios A2 y B2 con el paquete estadístico Excel 2016 para el balance hídrico mensual, con precipitaciones y cantidades de consumo obtenidas por Suastegui Cruz et al., (2018). La representación de los datos fue a través de gráficas de histogramas para el balance hídrico.

\section{Resultados}

Como se muestra en la figura 1, el balance hídrico anual de 1998 a 2016 proporciona un historial del comportamiento de la escasez de agua en el área de estudio. Se observa que los años más críticos fueron 2000, 2002, 2009, 2011, 2015 y 2016, esto basado en las actividades desarrolladas durante el periodo secano y de estiaje. Los años anteriores a 2012 mantuvieron una distribución constante entre las lluvias y el consumo de agua, pero aumentó en 2013 cuando ocurrieron las tormentas tropicales Ingrid y Manuel en Guerrero (Toscana Aparicio y Villaseñor Franco, 2018). En el año 2014 las lluvias fueron acompañaron por tormentas tropicales de menor intensidad, mientras que en 2015 y 2016 las precipitaciones fueron más bajas, por lo que causaron problemas de sequías en el periodo secano con pérdidas en la agricultura (Suastegui Cruz et al., 2018). 


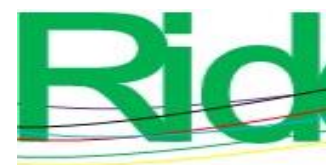

\section{Revista Iberoamericana para la Investigación y el Desarrollo Educativo ISSN $2007-7467$}

Figura 1. Balance hídrico anual de Las Animas Guerrero. para el periodo 1998-2016

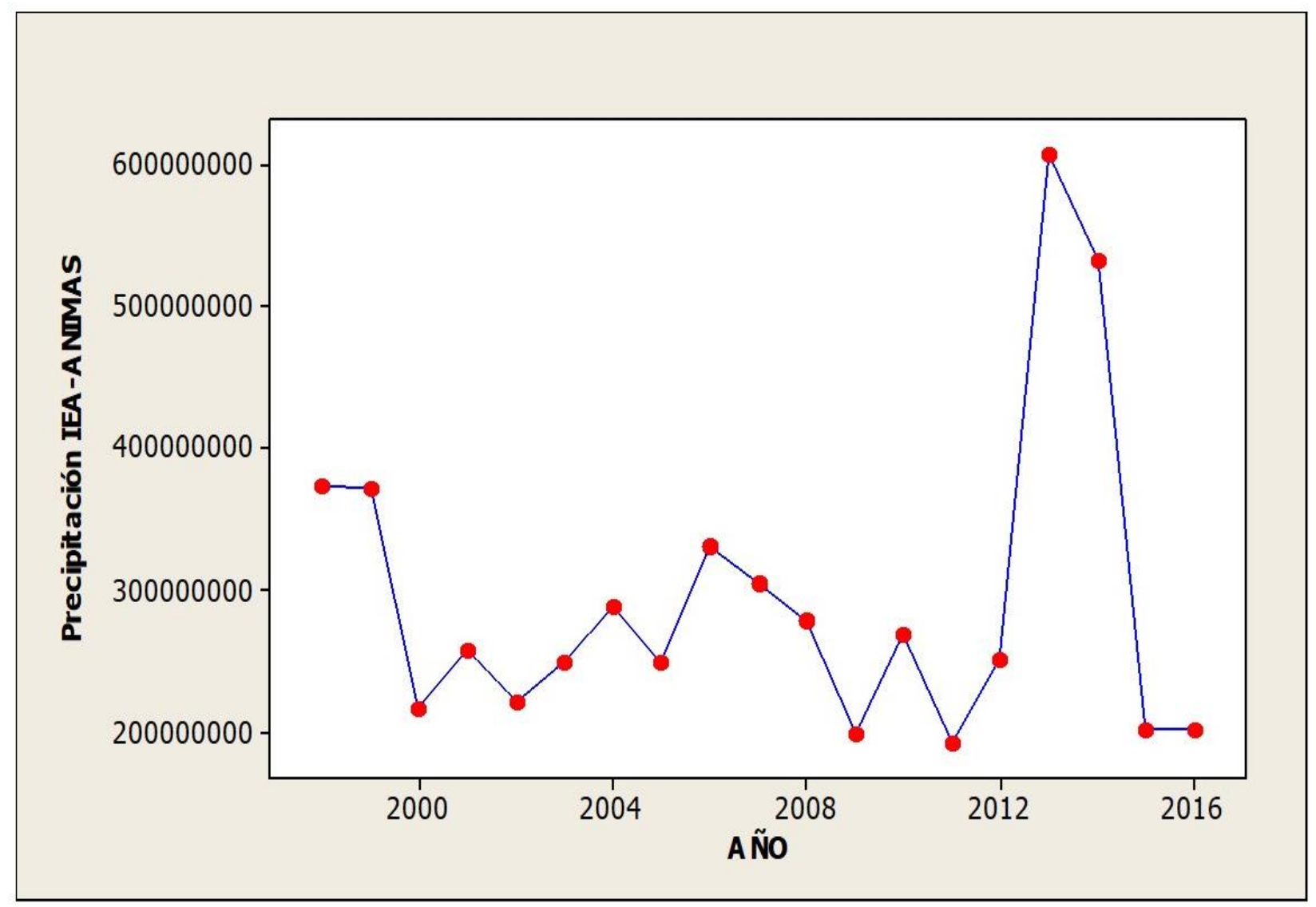

Fuente: Elaboración propia

Por otra parte, la proyección del balance hídrico mensual para el escenario A2 modelo MPI ECHAM 5 (figura 2) para el año 2030 muestra escasez de agua de aproximadamente 4, 000, 000, $000 \mathrm{~L} / \mathrm{ha}^{-1}$, pero con pronta recuperación en un periodo de 45 días. En los meses de mayo y junio de 2030 y 2050 aumentará la cantidad de agua, y permanecerá constante de junio a septiembre, en este sentido y de manera general, la disponibilidad de agua para el resto del año. Esto en comparación con el año 2017 que no presentó problemas del recurso agua en el periodo de estiaje. 


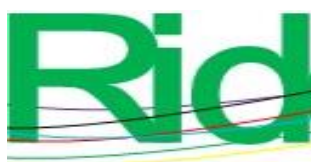

\section{Revista lberoamericana para la Investigación y el Desarrollo Educativo ISSN $2007-7467$}

Figura 2. Distribución del balance hídrico mensual de los años 2015, 2016, 2017 y del modelo MPI ECHAM 5 del escenario A2 de los años 2030 y 2050 de Las Animas Guerrero

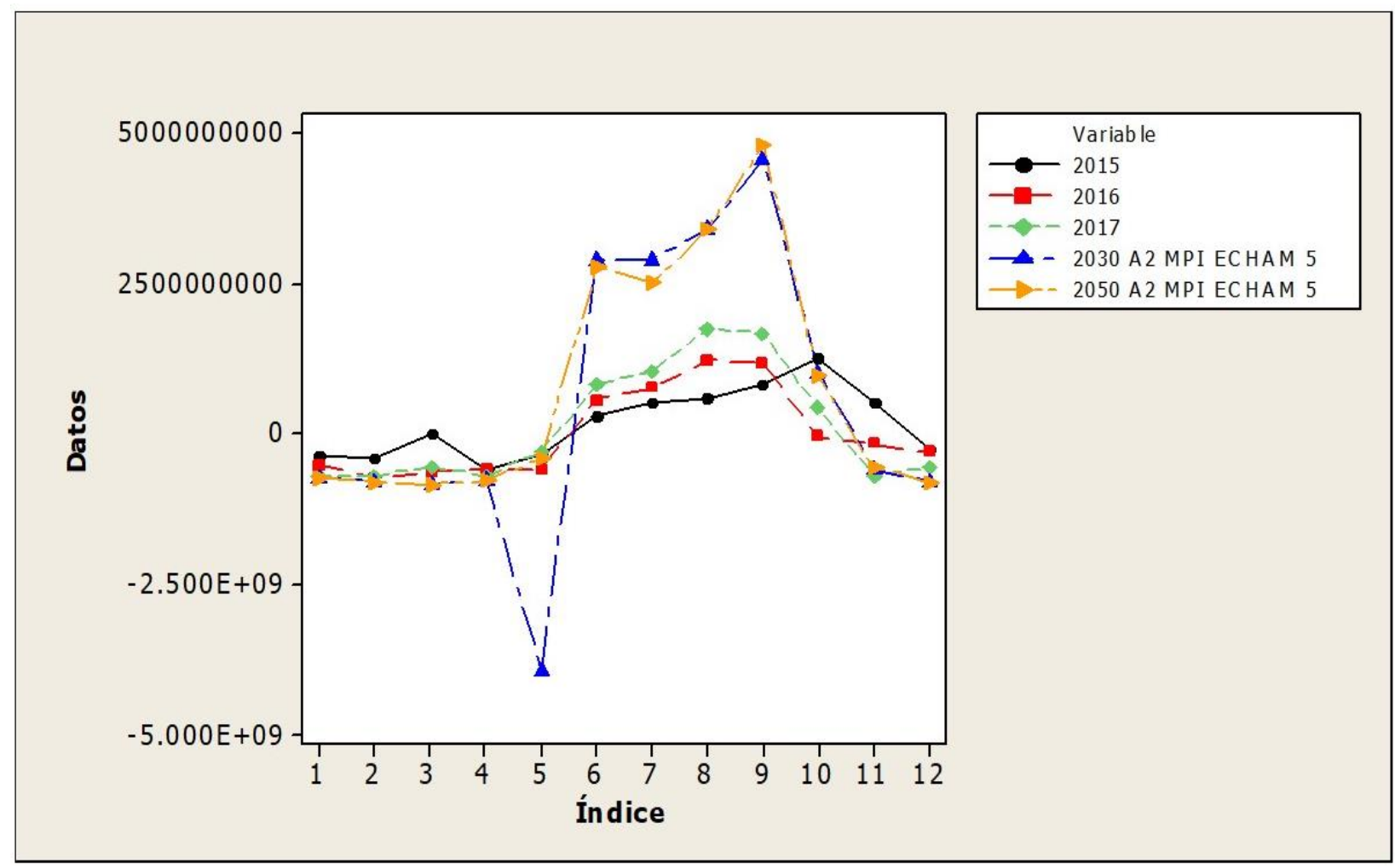

Fuente: Elaboración propia

La figura 3 muestra las modificaciones en la precipitación para el año 2030 que comenzará en el mes de junio y la estación de estiaje será de noviembre-junio con mayor duración en la localidad; esto con base en las proyecciones del escenario B2, modelo MPI ECHAM 5. Como resultado se tendrá que en el año 2030 y 2050 la precipitación será más abundante en el mes de septiembre, lo que coincide con el escenario A2. 


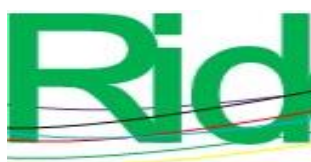

\section{Revista lberoamericana para la Investigación y el Desarrollo Educativo ISSN $2007-7467$}

Figura 4. Distribución del balance hídrico mensual de los años 2015, 2016, 2017 y del modelo HADGEM1 del escenario A2 de los años 2030 y 2050 de Las Animas Guerrero

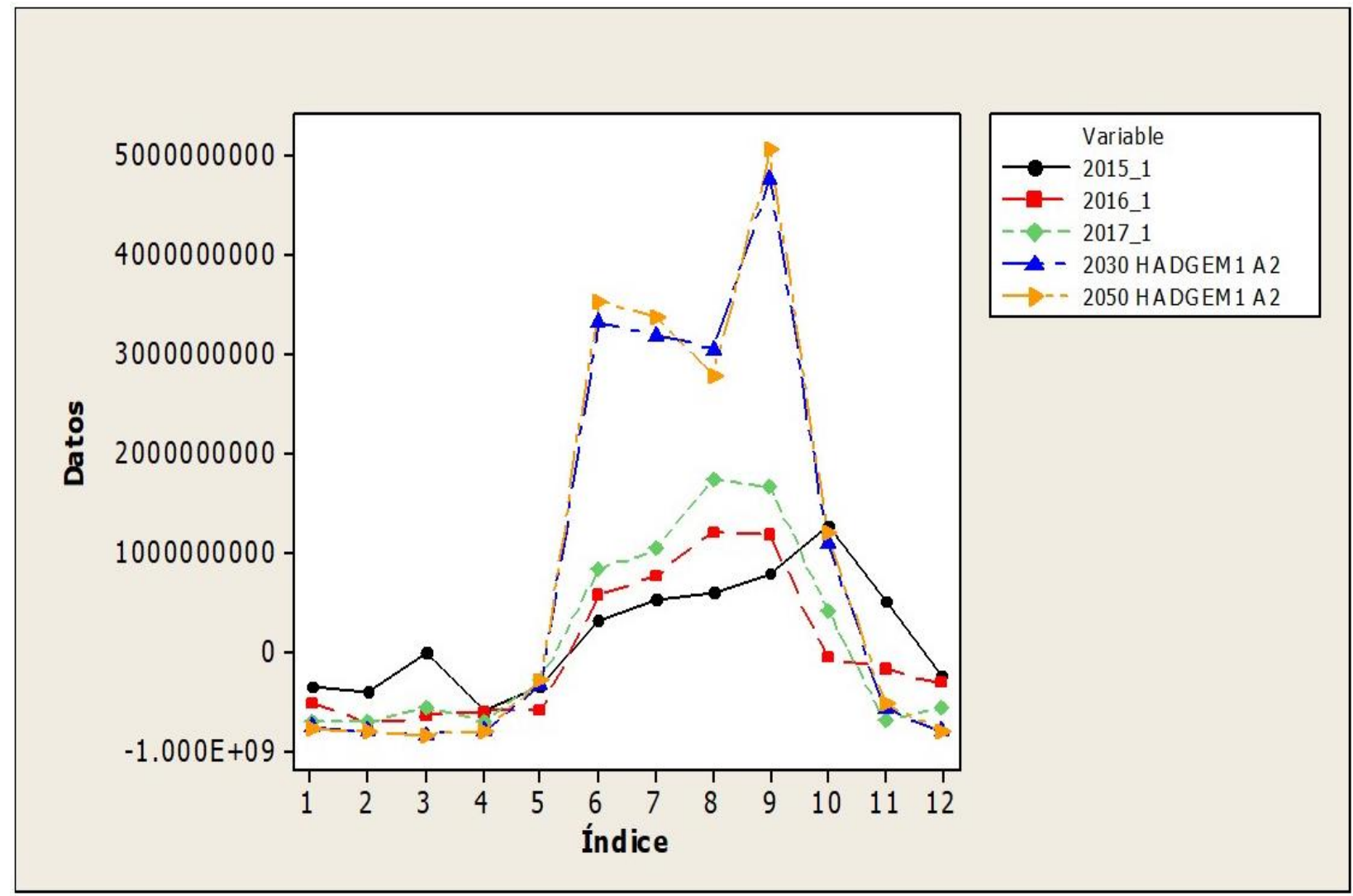

Fuente: Elaboración propia

Con los modelos de circulación, se puede visualizar que las lluvias futuras serán similares a las de años anteriores (figura 5), aunque la diferencia radica en los breves periodos de lluvias. Las predicciones de estos modelos permiten visualizar el cambio climático como uno de los principales contribuyentes a los efectos adversos durante las sequías en áreas agrícolas. 


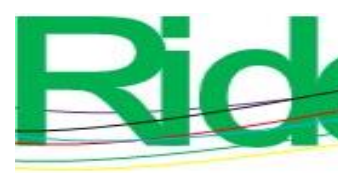

Revista Iberoamericana para la Investigación y el Desarrollo Educativo ISSN $2007-7467$

Se espera que el cambio climático continúe afectando las regiones y las condiciones climáticas regionales, lo que resultará en lluvias y sequías erráticas o irregulares (Suryabhagavan, 2017).

El aumento de las precipitaciones solo se dará por fenómenos como el Niño y la Niña, así como la situación presentada en 2013 (figura 1) que ocasionaron pérdidas en la agricultura por lluvias intensas (McNeeley et al., 2018), esto en contraste con Parry et al. (2007), quienes mencionan que la producción de alimentos en algunas regiones del mundo será estable para los años 2030 y 2050, mientras Hasegawa et al. (2018) proyectan un incremento en la inseguridad hídrica y alimentaria, con aumentos sustanciales de los precios y hambre en las regiones más pobres, que se combinarán con olas de calor durante las sequías y causarán daños a la agricultura, extinción de especies y escasez de agua (Esparza, 2014; McMaster et al., 2019; Miralles, Gentine, Seneviratne \& Teuling, 2019; Naumann et al., 2018; Sánchez-Balseca, Muñoz-Rodríguez y AldásSandoval, 2019).

Hoy en día, la seguridad hídrica se ve afectada por el cambio climático, lo que dificulta a los países que aún no han desarrollado estrategias hídricas para hacer frente a los procesos de adaptación (Flörke, Schneider \& McDonald, 2018; Sadoff y Muller, 2010).

Para Sadoff y Muller (2010) la seguridad hídrica se puede alcanzar solo si se invierte en las tres "I": a) información más accesible y adecuada, b) instituciones más fuertes y flexibles, y c) infraestructura natural y artificial para el almacenamiento, transporte y tratamiento del agua para la toma de decisiones. (p.5)

\section{Conclusiones}

De acuerdo con los escenarios para el cambio climático A2 y B2 para los años 2030 y 2050, con base en los modelos MPI ECHAM 5 y HadGEN1, en el territorio que marca a la comunidad de Las Ánimas, municipio de Tecoanapa Guerrero (México) se presentará mayor precipitación anual para los años 2030 y 2050 en comparación con los años 2015, 2016 y 2017. En tal sentido, se considera el mismo uso actual del agua por la comunidad donde el balance hídrico será superior, ocasionado por el incremento de las precipitaciones en periodos más cortos y con ampliación en periodos de estiaje. Por eso, es necesaria la búsqueda de mecanismo de resiliencia comunitaria anual con mejoras en la captación, conservación y manejo del agua para contribuir realmente a la seguridad hídrica y alimentaria local. 


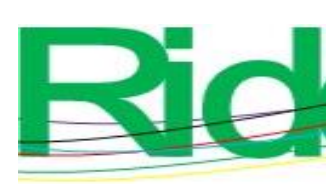

Revista Iberoamericana para la
Investigación y el Desarrollo Educativo
ISSN $2007-7467$

\section{Futuras líneas de investigación}

El presente estudio puede ser un referente para futuras investigaciones relacionadas con las ciencias ambientales o para aquellas que orientan sus líneas hacia problemáticas ambientales como el cambio climático, la escasez de agua y la producción de alimentos. En tal sentido, vale acotar que se han realizado investigaciones similares en países de Latinoamérica aunque escasos estudios en México, por lo que esta marcaría la pauta para comprender desde lo local cuáles serán las consecuencias que generará el cambio climático en las localidades rurales que desarrollan actividades agropecuarias en el periodo de lluvias. Cuando suelen suceder fenómenos se sequías de agua y alimentos existirán afectaciones económicas y sociales. Por ello, se sugiere orientar líneas de investigación de la percepción de los habitantes de zonas rurales sobre las consecuencias que les están generando las bajas precipitaciones en su entorno.

Existe inseguridad anualmente por parte de las personas sobre qué sucederá con el periodo de lluvias: si será un año bueno para la producción y si no habrá huracanes o lluvias intensas. Las prácticas inadecuadas que afectan al medio ambiente contribuyen a las modificaciones para los periodos de lluvias. El resultado y análisis comprendido marcó la pauta para sugerir que la población fomente la cultura de la prevención de riesgo y la ambiental, con el uso racional de sus recursos naturales, que a través de sus conocimientos empíricos tengan una conciencia para el cuidado y preservación de su entorno. 


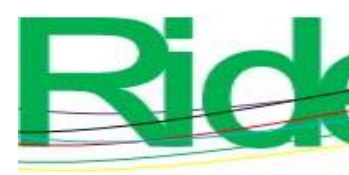

Revista Iberoamericana para la Investigación y el Desarrollo Educativo ISSN $2007-7467$

\section{Referencias}

Abou-Shaara, Hossam F. (2019). Geographical Information System for Beekeeping Development. Journal of Apicultural Science 63(1):5-16. doi: 10.2478/jas-2019-0015.

Agal'tseva, N. A., Tatjana Spectorman, C. J. White, \& T. W. Tanton. (2010). Modelling the future climate of the Amu Darya Basin. Interstate Water Resource Risk Management: Towards a sustainable future for the Aral Basin, IWA Publishing 9-32.

Alexander, Lisa V. (2016). Global Observed Long-Term Changes in Temperature and Precipitation Extremes: A Review of Progress and Limitations in IPCC Assessments and Beyond. Weather and Climate Extremes 11:4-16. doi: 10.1016/j.wace.2015.10.007.

de Amorim, Wellyngton Silva, Isabela Blasi Valduga, João Marcelo Pereira Ribeiro, Victoria Guazzelli Williamson, Grace Ellen Krauser, Mica Katrina Magtoto, \& José Baltazar Salgueirinho Osório de Andrade Guerra. (2018). The Nexus between Water, Energy, and Food in the Context of the Global Risks: An Analysis of the Interactions between Food, Water, and Energy Security. Environmental Impact Assessment Review 72:1-11. doi: 10.1016/j.eiar.2018.05.002.

Anderson, Martha C., Cornelio A. Zolin, Paulo C. Sentelhas, Christopher R. Hain, Kathryn Semmens, M. Tugrul Yilmaz, Feng Gao, Jason A. Otkin, \& Robert Tetrault. (2016). The Evaporative Stress Index as an Indicator of Agricultural Drought in Brazil: An Assessment Based on Crop Yield Impacts. Remote Sensing of Environment 174:82-99. doi: 10.1016/j.rse.2015.11.034.

Barnett, Jon, \& W. Neil Adger. (2007). Climate change, human security and violent conflict. Political geography 26(6):639-55.

Böhm, Reinhard, Philip D. Jones, Johann Hiebl, David Frank, Michele Brunetti, \& Maurizio Maugeri. (2010). The Early Instrumental Warm-Bias: A Solution for Long Central European Temperature Series 1760-2007. Climatic Change 101(1-2):41-67. doi: 10.1007/s10584-009-9649-4.

Buhaug, H. (2010). Climate Not to Blame for African Civil Wars. Proceedings of the National Academy of Sciences 107(38):16477-82. doi: 10.1073/pnas.1005739107.

Bussotti, Filippo, Martina Pollastrini, Vera Holland, \& Wolfgang Brüggemann. (2015). Functional Traits and Adaptive Capacity of European Forests to Climate Change. Environmental and Experimental Botany 111:91-113. doi: 10.1016/j.envexpbot.2014.11.006. 


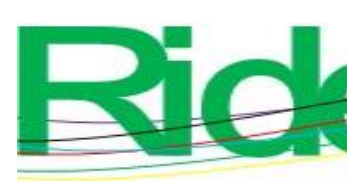

\section{Revista Iberoamericana para la Investigación y el Desarrollo Educativo ISSN 2007 - 7467}

Demuzere, M., M. Werner, N. P. M. van Lipzig, \& E. Roeckner. (2009). An Analysis of Present and Future ECHAM5 Pressure Fields Using a Classification of Circulation Patterns. International Journal of Climatology 29(12):1796-1810. doi: 10.1002/joc.1821.

Esparza, Miguel. (2014). La sequía y la escasez de agua en México: Situación actual y perspectivas futuras. Recuperado 27 de abril de 2020 (http://www.scielo.org.mx/scielo.php?pid=S018603482014000200008\&script=sci_arttext\&tlng=en).

Flörke, Martina, Christof Schneider, \& Robert I. McDonald. (2018). Water Competition between Cities and Agriculture Driven by Climate Change and Urban Growth. Nature Sustainability 1(1):51-58. doi: 10.1038/s41893-017-0006-8.

Frierson, Dargan M. W., Yen-Ting Hwang, Neven S. Fučkar, Richard Seager, Sarah M. Kang, Aaron Donohoe, Elizabeth A. Maroon, Xiaojuan Liu, \& David S. Battisti. (2013). Contribution of Ocean Overturning Circulation to Tropical Rainfall Peak in the Northern Hemisphere. Nature Geoscience 6(11):940-44. doi: 10.1038/ngeo1987.

García, E. (1973). Modificaciones al sistema de clasificación climática de Köppen. Instituto de Geografia. UNAM. Serie Libros No. 6.

Gleckler, Peter J., Paul J. Durack, Ronald J. Stouffer, Gregory C. Johnson, \& Chris E. Forest. (2016). Industrial-Era Global Ocean Heat Uptake Doubles in Recent Decades. Nature Climate Change 6(4):394-98. doi: 10.1038/nclimate2915.

Hagg, Wilfried, \& Christoph Mayer. (2016). Water of the Pamir - Potential and Constraints. Pp. 69-78 en Mapping Transition in the Pamirs: Changing Human-Environmental Landscapes, Advances in Asian Human-Environmental Research, editado por H. Kreutzmann y T. Watanabe. Cham: Springer International Publishing.

Hanjra, Munir A., \& M. Ejaz Qureshi. (2010). Global Water Crisis and Future Food Security in an Era of Climate Change. Food Policy 35(5):365-77. doi: 10.1016/j.foodpol.2010.05.006.

Hasegawa, Tomoko, Shinichiro Fujimori, Petr Havlík, Hugo Valin, Benjamin Leon Bodirsky, Jonathan C. Doelman, Thomas Fellmann, Page Kyle, Jason F. L. Koopman, Hermann Lotze-Campen, Daniel Mason-D’Croz, Yuki Ochi, Ignacio Pérez Domínguez, Elke Stehfest, Timothy B. Sulser, Andrzej Tabeau, Kiyoshi Takahashi, Jun'ya Takakura, Hans van Meijl, Willem-Jan van Zeist, Keith Wiebe, \& Peter Witzke. (2018). Risk of Increased Food Insecurity under Stringent Global Climate Change Mitigation Policy. Nature Climate Change 8(8):699-703. doi: 10.1038/s41558-018-0230-x.

Hernández-Mansilla, Alexis Augusto, Rogert Sorí-Gómez, Yadira Valentín-Pérez, Aliana LópezMayea, Orlando Córdova-García, y Oscar Benedico-Rodríguez. (2016). Sigatoka negra 


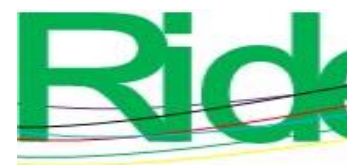

Revista Iberoamericana para la
Investigación y el Desarrollo Educativo
ISSN $2007-7467$

(Mycosphaerella fijiensis Morelet) y seguridad alimentaria. Escenarios bioclimáticos en bananos bajo efecto del cambio climático en Ciego de Ávila, Cuba. Journal of the Selva Andina Biosphere 4(2):59-70.

Hewitt, Chris D., Roger C. Stone, \& Andrew B. Tait. (2017). Improving the Use of Climate Information in Decision-Making. Nature Climate Change 7(9):614-16. doi: 10.1038/nclimate3378.

Instituto Nacional de Estadística y Geografía [INEGI]. (2010). Instituto Nacional de Estadística y Geografía. INEGI. Recuperado 27 de abril de 2020 (https://www.inegi.org.mx/). - Buscar con Google. https://www.google.com/search?q=INEGI\%2C+Instituto+Nacional+de+Estad\%C3\%ADs tica+y+Geograf\%C3\%ADa+(2010).+Instituto+Nacional+de+Estad\%C3\%ADstica+y+Ge ograf\%C3\%ADa.+INEGI.+Recuperado+27+de+abril+de+2020+(https\%3A\%2F\%2Fww w.inegi.org.mx \%2F).\&oq=INEGI\%2C+Instituto+Nacional+de+Estad\%C3\%ADstica+y+ Geograf\%C3\%ADa+(2010).+Instituto+Nacional+de+Estad\%C3\%ADstica+y+Geograf\% C3\%ADa.+INEGI.+Recuperado+27+de+abril+de+2020+(https\%3A\%2F\%2Fwww.inegi. org.mx\%2F).\&aqs=chrome..69i57.1308j0j15\&sourceid=chrome\&ie=UTF-8

Jimenez-Cisneros, Blanca. (2015). Seguridad Hídrica: Retos y respuestas, la fase VIII del Programa Hidrológico Internacional de la UNESCO (2014-2021). Revista Aqua-LAC 7(1):20-27.

Kang, Kiyoon, Sangkyu Park, Young Soon Kim, Sungbeom Lee, \& Kyoungwhan Back. (2009). Biosynthesis and Biotechnological Production of Serotonin Derivatives. Applied Microbiology and Biotechnology 83(1):27-34. doi: 10.1007/s00253-009-1956-1.

Kumar, Vijay, Sharad K. Jain, \& Yatveer Singh. (2010). Analysis of Long-Term Rainfall Trends in India. Hydrological Sciences Journal 55(4):484-96. doi: 10.1080/02626667.2010.481373.

McMaster, Gregory S., Debora A. Edmunds, Roger Marquez, Scott Haley, Gerald Buchleiter, Patrick Byrne, Timothy R. Green, Rob Erskine, Nathan Lighthart, Holm Kipka, Fred Fox, Larry Wagner, John Tatarko, Marc Moragues, \& Jim Ascough. (2019). Winter Wheat Phenology Simulations Improve When Adding Responses to Water Stress. Agronomy Journal 111(5):2350-60. doi: 10.2134/agronj2018.09.0615.

McNeeley, Shannon M., Candida F. Dewes, Crystal J. Stiles, Tyler A. Beeton, Imtiaz Rangwala, Michael T. Hobbins, \& Cody L. Knutson. (2018). Anatomy of an Interrupted Irrigation Season: Micro-Drought at the Wind River Indian Reservation. Climate Risk Management 19:61-82. doi: 10.1016/j.crm.2017.09.004. 

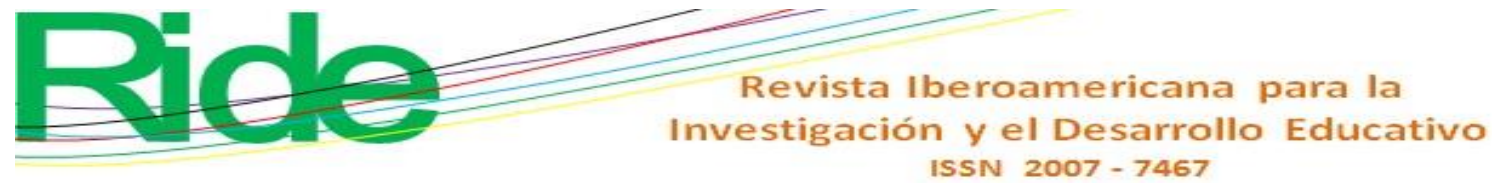

denitrification in wastewater using a biofilm reactor with rice shell as energy source. Tecnología y ciencias del agua 10(2):78-97. doi: 10.24850/j-tyca-2019-02-03.

Schubert, Siegfried D., Ronald E. Stewart, Hailan Wang, Mathew Barlow, Ernesto H. Berbery, Wenju Cai, Martin P. Hoerling, Krishna K. Kanikicharla, Randal D. Koster, Bradfield Lyon, Annarita Mariotti, Carlos R. Mechoso, Omar V. Müller, Belen Rodriguez-Fonseca, Richard Seager, Sonia I. Seneviratne, Lixia Zhang, \& Tianjun Zhou. (2016). Global Meteorological Drought: A Synthesis of Current Understanding with a Focus on SST Drivers of Precipitation Deficits. Journal of Climate 29(11):3989-4019. doi: 10.1175/JCLID-15-0452.1.

Seth, Anji, Sara A. Rauscher, Suzana J. Camargo, Jian-Hua Qian, \& J. S. Pal. (2007). RegCM3 Regional Climatologies for South America Using Reanalysis and ECHAM Global Model Driving Fields. Climate Dynamics 28(5):461-80. doi: 10.1007/s00382-006-0191-z.

Solomon, Susan, Martin Manning, Melinda Marquis, \& Dahe Qin. (2007). Climate change 2007the physical science basis: Working group I contribution to the fourth assessment report of the IPCC. Vol. 4. Cambridge university press.

Suastegui Cruz, Sirilo, Jose Luis Rosas Acevedo, Elias Hernandez Castro, América L. Rodríguez Herrera, y Maximino Reyes Umana. (2017). Caracterización del uso actual del suelo en Las Ánimas, municipio de Tecoanapa, Guerrero. Revista Iberoamericana de Ciencias 4(6):13243.

Suastegui Cruz, Sirilo, José Luis Rosas Acevedo, Maximino Reyes Umaña, América Libertad Rodríguez Herrera, Elías Hernández Castro, Felipe Gallardo López, \& Ana Patricia Leyva Zúñiga. 2018. Water Scarcity Index Calculation, Atlas Animas, Tecoanapa Municipality, Guerrero, Mexico. The Journal of Social Sciences Research 4(5):74-79.

Suryabhagavan, K. V. (2017). GIS-Based Climate Variability and Drought Characterization in Ethiopia over Three Decades. Weather and Climate Extremes 15:11-23. doi: 10.1016/j.wace.2016.11.005.

Toscana Aparicio, Alejandra, y Alma Villaseñor Franco. (2018). Las tormentas Ingrid y Manuel en La Montaña de Guerrero, 2013. La atención de la emergencia. Sociedad y ambiente (16):59-89.

Vörösmarty, C. J., P. B. McIntyre, M. O. Gessner, D. Dudgeon, A. Prusevich, P. Green, S. Glidden, S. E. Bunn, C. A. Sullivan, C. Reidy Liermann, \& P. M. Davies. (2010). Global Threats to Human Water Security and River Biodiversity. Nature 467(7315):555-61. doi: 10.1038/nature09440. 

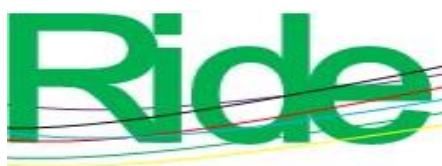

Revista Iberoamericana para la
Investigación y el Desarrollo Educativo
ISSN $2007-7467$

Wada, Yoshihide, \& Marc FP Bierkens. (2014). Sustainability of global water use: past reconstruction and future projections. Environmental Research Letters 9(10):104003.

Weber, Elke U. (2010). What Shapes Perceptions of Climate Change?: What Shapes Perceptions of Climate Change? Wiley Interdisciplinary Reviews: Climate Change 1(3):332-42. doi: $10.1002 /$ wcc. 41 .

Werner, M., B. Haese, X. Xu, X. Zhang, M. Butzin, \& G. Lohmann. (2016). Glacial-Interglacial Changes in H\&lt;Sub\&gt;2\&lt;/Sub\&gt;\&lt;Sup\&gt;18\&lt;/Sup\&gt;O, HDO and Deuterium Excess - Results from the Fully Coupled ECHAM5/MPI-OM Earth System Model. Geoscientific Model Development 9(2):647-70. doi: 10.5194/gmd-9-647-2016.

Xin, Yufei, Yongjiu Dai, Jian Li, Xinyao Rong, \& Guo Zhang. (2019). Coupling the Common Land Model to ECHAM5 Atmospheric General Circulation Model. Journal of Meteorological Research 33(2):251-63. doi: 10.1007/s13351-019-8117-y.

Zhang, Chi, Xiaoxian Chen, Yu Li, Wei Ding, \& Guangtao Fu. (2018). Water-Energy-Food Nexus: Concepts, Questions and Methodologies. Journal of Cleaner Production 195:625-39. doi: 10.1016/j.jclepro.2018.05.194. 